\title{
Reference Measurement Procedure Development for C-Reactive Protein in Human Serum
}

\author{
Eric L. Kilpatrick*,+ and David M. Bunk ${ }^{\star}$
}

\begin{abstract}
National Institute of Standards and Technology, Chemical Sciences Technology Laboratory, Analytical Chemistry Division, 331 Fort Johnson Road, Charleston, South Carolina 29412, and National Institute of Standards and Technology, Chemical Sciences Technology Laboratory, Analytical Chemistry Division, 100 Bureau Drive, Gaithersburg, Maryland 20899
\end{abstract}

This paper describes the development of a reference measurement procedure to quantify human C-reactive protein (CRP) in serum using affinity techniques prior to tryptic digestion and liquid chromatography- tandem mass spectrometry (LC-MS/MS) for the certification of reference materials in clinically relevant ranges. The absence of a suitable internal standard for the CRP measurement, necessary to eliminate potential measurement bias in both the affinity purification and trypsin digestion steps, was addressed using the method of standard addition. The standard addition quantification approach was combined with affinity purification, using an anti-CRP monoclonal antibody conjugated to polystyrene beads, trypsin digestion of the purified protein, and LC-MS/MS analysis of CRP tryptic peptides. The effectiveness of intact protein affinity purification was evaluated through the measurement of CRP in several serum-based CRP control materials, yielding levels that were comparable to their expected mean concentration values. Quantitative results were confirmed with an external calibration approach. This study demonstrates the feasibility of affinity purification with LC-MS/MS for the reference measurement procedure development of low abundance serum protein analytes.

Reference measurement procedures are used for a variety of purposes including (1) the assessment of the measurement quality of routine measurement procedures, (2) the value assignment of high-order calibration solutions (the so-called "master calibrators") for routine assays, and (3) the value assignment of certified reference materials. ${ }^{1,2}$ In these roles, reference measurement procedures are considered "higher-order" measurement procedures, as they aim to achieve a higher level of accuracy and precision than the routine assay for the analyte being measured, producing a value with a low uncertainty of defined magnitude. In order for a reference measurement procedure to be fit for this purpose, it must be thoroughly evaluated to identify sources of

\footnotetext{
* Corresponding author. Telephone: 843-725-4819, fax: 843-762-8742, e-mail: eric.kilpatrick@nist.gov.

${ }^{\dagger}$ National Institute of Standards and Technology, Charleston, SC.

₹ National Institute of Standards and Technology, Gaithersburg, MD.

(1) Vitzthum, F.; Siest, G.; Bunk, D. M.; Preckel, T.; Wenz, C.; Hoerth, P.; Schulz-Knappe, P.; Tammen, H.; Adamkiewics, J.; Merlini, G.; Anderson, N. L. Proteomics Clin. Appl. 2007, 1, 1016-1035.

(2) Bunk, D. M. Clin. Biochem. Rev. 2007, 28, 131-137.
}

bias and assess their magnitude. ${ }^{3-5}$ Minimizing or eliminating bias is necessary to measure the most accurate, true concentration of the analyte. Because of the need for high levels of accuracy and precision, reference measurement procedures are typically low-throughput, labor intensive, and often costly to perform, in sharp contrast to what is desirable for routine measurements.

Reference measurement procedures are key elements in the establishment of measurement standardization and metrological traceability in clinical chemistry. For more than 30 years, isotope dilution mass spectrometry (IDMS) has been one of the analytical techniques used frequently in reference measurement procedures, particularly for small molecule and inorganic analytes of clinical importance. ${ }^{6}$ Despite this, few reference measurement procedures exist for clinically relevant proteins. This is one reason why so many clinical protein analytes are measured in arbitrary International Units (IUs); without a reference measurement procedure to establish metrological traceability, measurement of true concentrations, those linked accurately to Système International (SI) units such as the mole, is not possible and arbitrary units are often the only option for the value-assignment of reference materials and calibrators.

Because IDMS is used so extensively in reference measurement procedures for many clinical analytes, it seems natural to use this technique in reference measurement procedures for clinically relevant proteins. Unfortunately, the application of IDMS to protein measurements is not straightforward within the measurement constraints of reference measurement procedures due to the lack of suitably labeled intact proteins. As a substitute for intact labeled proteins, the use of isotopically labeled peptides as internal standards in MS-based quantitative proteomics measurements is widely practiced. ${ }^{7-11}$ The isotopically labeled peptides in quantitative proteomics applications act as internal

(3) ISO Guide 35:2006 Reference Materials - General and staticstical principles for certification; ISO, Geneva, Switzerland, 2006.

(4) NIST Special Publication 260-136 Definitions of Terms and Modes Used at NIST for Value-Assignment of Reference Materials for Chemical Measurements; U.S. Government Printing Office, Washington, D.C., 2000.

(5) ISO 15193:2002 In vitro diagnostic medical devices - Measurement of quantities in samples of biological origin - Presentation of reference measurement procedures; ISO, Geneva, Switzerland, 2002.

(6) Copeland, B. E.; Grisley, D. W.; Casella, J.; Bailey, H. Am. J. Clin. Pathol. 1976, 66, 619-633.

(7) Barr, J. R.; Maggio, V. L.; Patterson, D. G., Jr.; Cooper, G. R.; Henderson, L. O.; Turner, W. E.; Smith, S. J.; Hannon, W. H.; Needham, L. L.; Sampson, E. J. Clin. Chem. 1996, 42, 1676-1682.

(8) Gerber, S. A.; Rush, J.; Stemman, O.; Kirschner, M. W.; Gygi, S. P. Proc. Natl. Acad. Sci. U.S.A. 2003, 100, 6940-6945. 
standards only during the LC-MS/MS measurement but not during prior sample preparation such as enzymatic proteolysis or abundant protein depletion. These sample preparation steps are potential sources of bias, the magnitude of which is unknown and potentially variable from analysis to analysis making the determination of uncertainty problematic. In IDMS-based reference measurement procedures for small organic molecules, readily available ${ }^{13} \mathrm{C}$ - or deuterium-labeled analogues of the analyte are added to the sample immediately, at the start of the analysis, prior to all sample preparation steps. For proteins, the equivalent approach would be to use a ${ }^{15} \mathrm{~N}$-labeled protein, obtained through molecular biological approaches, as the ideal internal standard that would be present during all stages of sample preparation and analysis and behavior chemically equivalently to the analyte protein. ${ }^{12-14}$ Unfortunately, for many clinically relevant proteins, producing a ${ }^{15} \mathrm{~N}$-labeled recombinant version that has the same post-translational modifications and protein-protein interactions as the analyte protein is a significant technical challenge, often not practical or feasible.

The method of standard addition is commonly used in analytical chemistry when appropriate internal standards for the analyte are not available and has been successfully used in proteomic measurements. ${ }^{15-17}$ In standard addition, an additional quantity of the analyte is spiked directly to the sample, augmenting the endogenous analyte concentration. The increase in the analytical signal of the spiked sample relative to the unspiked sample can be used to determine the endogenous concentration of analyte. If the sample preparation and analysis of the spiked and unspiked samples are carried out identically, calibration is obtained directly without an internal standard. For protein analytes in which a purified protein standard is available for spiking, the method of standard addition may allow protein quantification to be achieved without bias from sample preparation and may be a viable alternative to the use of an isotopically labeled internal standard in reference measurement procedures for proteins.

To investigate whether standard addition could be used successfully to quantify a clinically relevant protein in a complex serum matrix, we applied this method to the quantification of human C-reactive protein (CRP). We combined the standard addition of purified CRP to serum samples with quantitative proteomics methodology in which the spiked-serum sample is proteolyzed with trypsin and tryptic peptides are quantified using LC-MS/MS with multiple reaction monitoring (MRM). Although the measurement sensitivity of MRM-based LC-MS/MS is sufficient to measure normal serum CRP concentrations $(<1 \mathrm{mg} / \mathrm{L})$,

(9) Barnidge, D. R.; Goodmanson, M. K.; Klee, G. G.; Muddiman, D. C. J. Proteome Res. 2004, 3, 644-652.

(10) Aguiar, M.; Masse, R.; Gibbs, B. F. Anal. Biochem. 2006, 354, 175-181.

(11) Arsene, C. G.; Ohlendorf, R.; Burkitt, W.; Pritchard, C.; Henrion, A. O'Connor, G.; Bunk, D. M.; Guttler, B. Anal. Chem. 2008, 80, 4154-4160.

(12) Van Uytfanghe, K.; Rodriquez-Cabaleiro, D.; Stockl, D.; Thienpont, L. M. Rapid Commun. Mass Spectrom. 2007, 21, 819-821.

(13) Singh, R.; Crow, F. W.; Babic, N.; Lutz, W. H.; Lieske, J. C.; Larson, T. S.; Kumar, R. Clin. Chem. 2007, 53, 540-542.

(14) Seegmiller, J. C.; Barnidge, D. R.; Burns, B. E.; Larson, T. S.; Lieske, J. C.; Kumar, R. Clin. Chem. 2009, 55, 1100-1107.

(15) Nelson, R. W.; McLean, M. A.; Hutchens, T. W. Anal. Chem. 1994, 66, 1408-1415.

(16) Mayr, B. M.; Kohlbacher, O.; Reinert, K.; Sturm, M.; Gropl, C.; Lange, E.; Klein, C.; Huber, C. G. J. Proteome Res. 2006, 5, 414-421.

(17) Bondar, O. P.; Barnidge, D. R.; Klee, E. W.; Davis, B. J.; Klee, G. G. Clin. Chem. 2007, 53, 673-678. we included immunoenrichment, using an anti-CRP monoclonal antibody coupled to magnetic beads, to the sample preparation in order to achieve a higher analytical signal response for maximal measurement precision.

CRP is a circulating protein in the blood which has been investigated as a clinical diagnostic marker and was chosen for investigation because it is stable, commercial monoclonal antibodies are available, and it is of clinical interest. A number of disease states have been correlated with increased levels of serum CRP including cardiovascular disease and systemic inflammatory conditions. CRP has been recently described as an independent marker for coronary heart disease with a stratification corresponding to low $(<1 \mathrm{mg} / \mathrm{L})$, moderate $(1 \mathrm{mg} / \mathrm{L}$ to $3 \mathrm{mg} / \mathrm{L})$, and high $(>3 \mathrm{mg} /$ L) levels of cardiovascular risk. ${ }^{18}$ Currently, there are no certified reference materials having CRP concentrations at meaningful levels for cardiovascular clinical analyses. The clinical measurement of CRP for the determination of cardiovascular risk is performed by use of high sensitivity CRP nephelometry/immunoturbidimetric assays which are generally value-assigned based upon recognized certified reference material (ERM DA472, plasma proteins in human serum) issued by the Institute for Reference Materials and Measurements. This reference material contains $\mathrm{CRP}$ with an assigned concentration of $0.0418 \mathrm{~g} / \mathrm{L}( \pm 0.0025 \mathrm{~g} / \mathrm{L})$ which is greater than 10-fold higher than moderate levels in serum. Generation of suitable calibrants for CRP measurement of either normal or moderately elevated CRP levels require the local dilution of ERM DA472, which raises commutability and standardization issues. ${ }^{19}$

The purpose of this study was to evaluate the combination of standard addition, affinity purification, and MRM-based LC-MS/ MS quantification of tryptic peptides to develop an accurate and precise reference measurement procedure for $\mathrm{CRP}$ in serum. The method could be applied equally well for the analyte of any compound (protein, peptide, or small molecule) for which a proven antibody exists.

\section{MATERIALS AND METHODS}

Disclaimer: Certain commercial equipment, instruments, and materials are identified in this paper to adequately specify the experimental procedure. Such identification does not imply recommendation or endorsement by NIST nor does it imply that the equipment, instruments, or materials are necessarily the best available for the purpose.

Materials. Acetonitrile-HPLC grade and trifluoroacetic acid (TFA) were obtained from Burdick \& Jackson (Muskegon, MI). Water-LC/MS grade was purchased from J.T. Baker (Phillipsburg, $\mathrm{NJ}$ ). Rapigest SF was purchased from Waters Corporation (Milford, MA). Modified sequencing grade trypsin was obtained from Promega (Madison, WI). Dynabeads MyOne Tosylactivated superparamagnetic polystyrene beads were purchased from Invitrogen (Carlsbad, CA). CRP monoclonal antibody (clone 3) was obtained from Meridian Life Science (Saco, ME). High purity C-reactive protein from human serum was obtained from EMD

(18) Pearson, T. A.; Mensah, G. A.; Alexander, R. W.; Anderson, J. L.; Cannon, R. O., III; Criqui, M.; Fadl, Y. Y.; Fortmann, S. P.; Hong, Y.; Myers, G. L.; Rifai, N.; Smith, S. C., Jr.; Taubert, K.; Tracy, R. P.; Vinicor, F. Circulation 2003, 107, 499-511.

(19) Johnson, A. M.; Ledue, T. B.; Collins, M. F. Clin. Chem. Lab. Med. 2003, 4, 177-182. 
Biosciences, Inc. (La Jolla, CA). High-Sensitive CRP control serum was purchased from Kamiya Biomedical (Seattle, WA) as well as ASO/RF/CRP control serum. Isotopically labeled and unlabeled versions of the synthetic peptides, GYSIFSYATK (monoisotopic mass of $1136 \mathrm{Da}$ ) and YEVQGEVFTKPQLWP (monoisotopic mass of $1820 \mathrm{Da}$ ) were purchased from Anaspec, Inc. (San Jose, CA). The labeled version of the peptides were synthesized with ${ }^{13} \mathrm{C}_{9},{ }^{15} \mathrm{~N}_{1}$-phenylalanine (GYSIFSYATK) or ${ }^{13} \mathrm{C}_{5},{ }^{15} \mathrm{~N}_{1}$-valine for each valine residue (YEVQGEVFTKPQLWP) (purity $>98 \%$ ). Other reagents and materials were obtained from SigmaAldrich (St. Louis, MO).

Affinity Purification of CRP. CRP mAb was conjugated to polystyrene superparamagnetic beads according to vendor protocol and further described in Supporting Information. CRP mAbconjugated beads $(15 \mu \mathrm{L})$ were placed into $1.5 \mathrm{~mL}$ centrifuge tubes, and the supernatant was removed by magnetic separation. PBS buffer supplemented with $1 \mathrm{~g} / \mathrm{L}$ BSA and $0.5 \mathrm{~mL} / \mathrm{L}$ Tween20 was added in quantity sufficient to achieve a volume of $500 \mu \mathrm{L}$ after the addition of CRP-containing solutions. After $2 \mathrm{~h}$ incubation (room temperature) with rotation, beads were rinsed twice with $1 \mathrm{~mL}$ of PBS supplemented with $1 \mathrm{~g} / \mathrm{L}$ BSA followed by two rinses with water supplemented with $1 \mathrm{~g} / \mathrm{L}$ BSA. After removal of the last rinse, $40 \mu \mathrm{L}$ of elution buffer $(100 \mathrm{~mL} / \mathrm{L}$ acetonitrile, $4 \mathrm{~mL} / \mathrm{L}$ TFA with $1.67 \mathrm{~g} / \mathrm{L}$ BSA) was added to the beads which were briefly vortexed. The eluted CRP was stored frozen $\left(-20^{\circ} \mathrm{C}\right)$ until digestion.

Method of Standard Addition for CRP Quantification. Affinity purifications were performed on control serum having two levels of CRP in separate sets. Set A (ASO/RF/CRP Control Level 2 serum, nominal $38 \mathrm{mg} / \mathrm{L} \mathrm{CRP)} \mathrm{and} \mathrm{Set} \mathrm{B} \mathrm{(High-Sensitive} \mathrm{CRP}$ Control Level 1, nominal $0.5 \mathrm{mg} / \mathrm{L}$ ) were quantified by one-point standard addition. Serum was added to the prepared beads in buffer to achieve an approximately 20-fold and 2-fold dilution for the high and low serum sets, respectively, in a $500 \mu \mathrm{L}$ reaction volume. The CRP standard was diluted to $0.1 \mu \mathrm{g} / \mu \mathrm{L}$ from the stock concentration of $6.1 \mu \mathrm{g} / \mu \mathrm{L}$ (as determined by the manufacturer) by addition of PBS supplemented with $1 \mathrm{~g} / \mathrm{L}$ BSA, which pilot experiments found to increase $\mathrm{CRP}$ detection and quantification, presumably by blocking nonspecific losses of CRP protein and peptides to the pipet tips and tubes. CRP standard protein was spiked in to produce 0 and $3 \mu \mathrm{g}$ addition levels for quantification purposes. Additional spikes were performed at 1 and $2 \mu \mathrm{g}$ levels to confirm linearity. The standard addition affinity purifications were conducted on three separate days within a span of four days. Affinity purification of the high CRP serum set was performed on days one and three and included replicates A1, A2, A3 and A4, $A 5, A 6$, respectively. Day four purifications were performed using the low CRP serum set with three replicates (B1, B2, and B3). An additional set of purifications was performed on days one and four to generate an external reference curve using only the CRP standard in aqueous solution containing $0.1,1,3$, and $5 \mu \mathrm{g}$ of added CRP standard.

As a comparison, a second standard addition analysis was performed without the affinity purification step. The CRP standard was spiked into high and low CRP serum samples immediately prior to denaturation, alkylation, and digestion with trypsin and subsequent LC-MS/MS analysis.
Digestion of Protein. Samples were reduced to dryness using a vacuum centrifuge at $30^{\circ} \mathrm{C}$ and reconstituted in $50 \mu \mathrm{L}$ of 50 $\mathrm{mmol} / \mathrm{L}$ Tris $(\mathrm{pH} 8.3)$ containing $5 \mathrm{mmol} / \mathrm{L}$ DTT and $2 \mathrm{~g} / \mathrm{L}$ Rapigest. The tubes were heated to $99.5^{\circ} \mathrm{C}$ for $5 \mathrm{~min}$ and then kept at $60^{\circ} \mathrm{C}$ for $30 \mathrm{~min}$. Upon cooling and gentle vortexing, 15 $\mathrm{mmol} / \mathrm{L}$ of iodoacetamide was added and incubated in the dark $(1 \mathrm{~h})$. The alkylation reaction was stopped with the addition of DTT to reach $21 \mathrm{mmol} / \mathrm{L}$. A sufficient quantity of $50 \mathrm{mmol} / \mathrm{L}$ Tris (pH 8.3) and $5 \mathrm{mmol} / \mathrm{L}$ DTT was added to reduce the Rapigest concentration to $1 \mathrm{~g} / \mathrm{L}$ in a $100 \mu \mathrm{L}$ reaction volume. Trypsin was freshly reconstituted $(1 \mu \mathrm{g} / \mu \mathrm{L})$ in provided buffer and added to the samples $(60: 1(\mathrm{~m} / \mathrm{m})$ protein-to-trypsin ratio). The tubes were incubated for $45-48 \mathrm{~h}$ at $37^{\circ} \mathrm{C}$ with shaking. A second application of trypsin was added after $24 \mathrm{~h}$ to ensure complete digestion. The digestion was stopped with the addition of $0.5 \mu \mathrm{L}$ of TFA and incubated at $37^{\circ} \mathrm{C}$ for $1 \mathrm{~h}$ prior to centrifugation and removal of the supernatant for analysis. The digestion products were stored frozen $\left(-20{ }^{\circ} \mathrm{C}\right)$ until analysis.

LC-MS/MS Analysis. The digestion products were thawed, and $80 \mu \mathrm{L}$ was transferred to fresh $1.5 \mathrm{~mL}$ centrifuge tube and then reduced to dryness prior to the addition of $30 \mu \mathrm{L} \mathrm{LC} / \mathrm{MS}$ grade water containing labeled peptides $(130 \mathrm{pmol})$ to confirm the identity of eluted peptides. The labeled peptides and digested samples were equilibrated at room temperature for $45 \mathrm{~min}$ before transfer to autosampler vials. Each analysis set was prepared for analysis as one lot and reconstituted using the same preparation of labeled peptides and run as one LC-MS/MS batch using Applied Biosystems API 4000 with an Agilent 1100 LC. Details of the LCMS/MS analysis are provided in the Supporting Information. The response areas for each peptide were combined for the quantitative analysis.

\section{RESULTS}

Characterization of CRP Affinity Purification Reagents. The CRP standard must behave as identically to the native serum $\mathrm{CRP}$ as possible in order to minimize potential heterogeneity bias. This study made use of commercially available purified human serum CRP to achieve fidelity to the analyte to characterize the CRP mAb-conjugated beads and to use as the standard addition spike. The purity of the CRP standard was described as $100 \%$ by the vendor according to SDS-PAGE analysis and was further analyzed by MALDI-MS in the present study with details of the analysis method, along with its suitability for use in affinity purification, provided in the Supporting Information (Figures S-1 and S-2). Figure 1 shows the spectra from MALDI-MS analysis of the elution product of the affinity purification of intact endogenous CRP from human serum. The nominal CRP content of the serum was $11 \mathrm{mg} / \mathrm{L}$, and a peak is observed at the appropriate mass/charge ratio $(m / z)$ for the singly charged protomer form of CRP consistent with that seen with CRP standard confirming the identity of the intact affinity-purified protein as endogenous CRP. These results demonstrate that the CRP mAb-conjugated beads can effectively capture the CRP analyte from serum.

Quantitative analysis of CRP was not performed on the intact affinity-purified protein but on peptides derived from the trypsin digestion of CRP. A representative LC-MS/MS chromatogram is shown in Figure 2, overlaying the MRM signals for two "signature" peptides (with monoisotopic molecular masses of 1136 and 1820 Da respectively) observed in the tryptic digest of affinity purified 


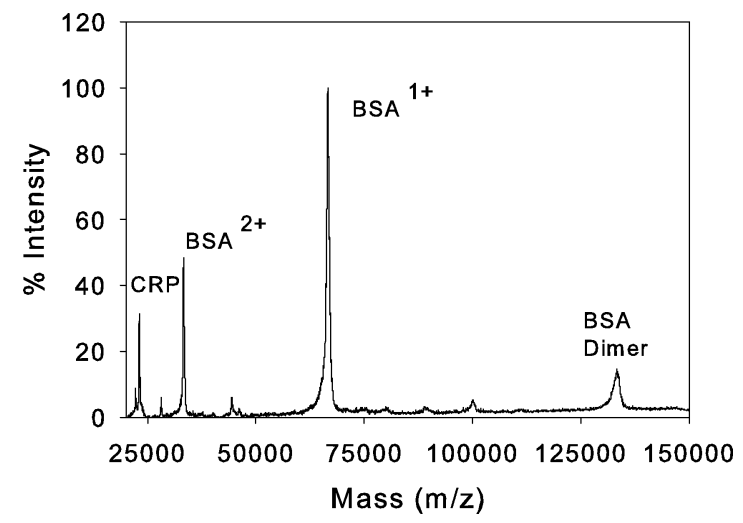

Figure 1. Mass spectra from MALDI-MS analysis of endogenous CRP from serum. Spectra were acquired of a sample which had undergone affinity purification of the intact endogenous CRP from $250 \mu \mathrm{L}$ of human serum control containing $\approx 11 \mu \mathrm{g}$ CRP. BSA is added during the affinity purification elution to decrease nonspecific binding.

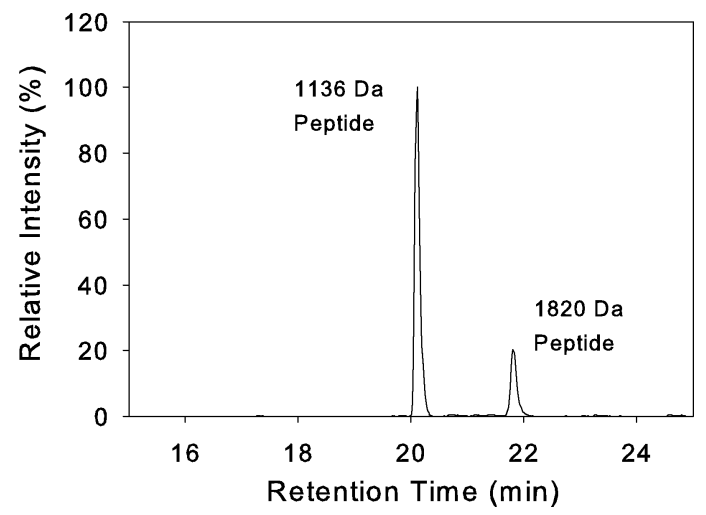

Figure 2. LC-MS/MS spectra of peptides from CRP affinity purification from serum. Spectra are shown for the LC-MS/MS analysis of a tryptic digestion of CRP which has been affinity purified from serum containing $\approx 38 \mathrm{mg} / \mathrm{L}$ CRP. Two peptides were monitored for quantification, GYSIFSYATK (1136 Da, ₹20.2 min retention), and YEVQGEVFTKPQLWP (1820 Da, $\approx 21.8 \mathrm{~min}$ retention). Data are normalized to the peak corresponding to the 1136 Da peptide in each set.

CRP from serum with a nominal $38 \mathrm{mg} / \mathrm{L}$ CRP concentration. Identity of the correct peptide was corroborated by the coincident elution of the synthetic labeled form of each respective peptide (data not shown).

The CRP mAb-conjugated beads were further assessed by affinity purification of aqueous solutions of CRP standard followed by trypsin digestion and LC-MS/MS analysis. The binding characteristics of the $\mathrm{CRP} \mathrm{mAb}$-conjugated beads were evaluated by analysis of the response of CRP captured after affinity purification from aqueous solutions of CRP standard at 1, 3, 5, 10 , and $20 \mu \mathrm{g}$ levels in duplicate. Figure 3 illustrates linear binding in the range from $1 \mu \mathrm{g}$ to $5 \mu \mathrm{g}\left(R^{2}>0.98\right)$ with a saturation limit between $5 \mu \mathrm{g}$ and $10 \mu \mathrm{g}$. Therefore, affinity purifications in the study were performed limiting the amount of CRP in each reaction to less than $5 \mu \mathrm{g}$. These results are significant in demonstrating that the binding of the CRP to the CRP mAbconjugated bead occurs in a manner consistent with classical principles of receptor-ligand binding mechanisms. Steady-state binding of CRP to the beads was achieved at the time used in the study (2 h), as no significant differences in the LC-MS/

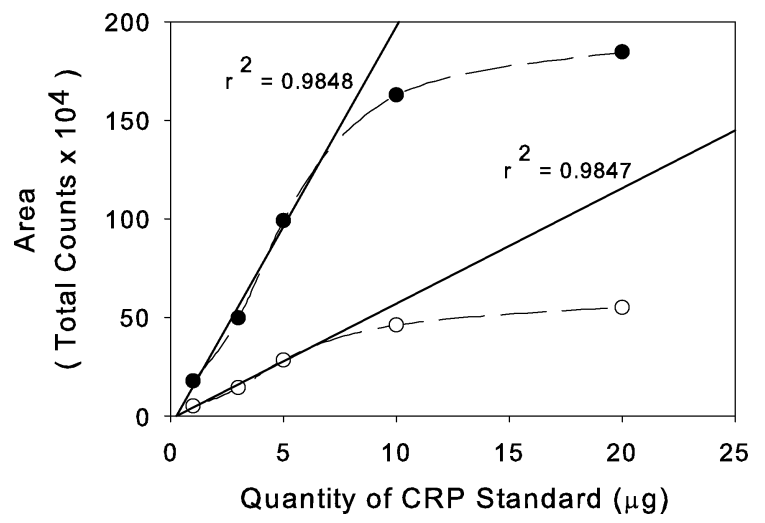

Figure 3. Concentration-response of affinity purification of CRP standard. CRP standard was affinity purified in duplicate from aqueous buffer at five levels (1, 3, 5, 10, and $20 \mu \mathrm{g})$ and digested with trypsin before analysis by LC-MS/MS. The mean area at each level is shown for the GYSIFSYATK, $1136 \mathrm{Da}$ (solid circles), and YEVQGEVFTKPQLWP, 1820 Da (open circles), peptides at each level. The linear regression parameters for the first three levels are shown next to each respective line.

MS response were seen at different affinity purification incubation times $(1,2,4,6$, and 8$) \mathrm{h}$ (data not shown).

The influence of nonspecific binding of CRP during affinity purification was investigated using a control set of beads in which BSA was conjugated in place of CRP. CRP standard ( $20 \mu \mathrm{g}, 5$ fold increase over typical) was incubated with the control beads beside a parallel incubation of CRP mAb-conjugated beads. LC-MS/MS analysis of the resulting trypsin digestion indicated that nonspecific binding of CRP is negligible, reaching a level of only $0.1 \%$ of maximum peak intensity (data not shown).

Inconsistency in the tryptic digestion of the affinity purified protein could add considerable bias to the measurement result by influencing the linearity of the affinity purified measurand response by LC-MS/MS. To investigate the contribution of enzymatic digestion to error of the measurement, increasing amounts of CRP standard were prepared in elution buffer and digested in triplicate and analyzed by LC-MS/MS. The results in Supporting Information Figure S-3 show that the release of signature peptides from tryptic digestion is linear across the range of $0.1 \mu \mathrm{g}$ to $10 \mu \mathrm{g}$ for each peptide with values of $r^{2}>0.99$. Although the efficiency of the digestion cannot be determined from this analysis, it does demonstrate the linearity of the tryptic digestion in this study. Therefore, digestion of the total amount of CRP (endogenous and spiked) in a sample can be assumed to be uniformly digested by trypsin. Therefore, the CRP added to the sample functions to remove bias so that tryptic digestion contributes only random error to the measurement analysis.

An estimate of the percentage of CRP which has been bound to and eluted from the beads can be made by comparing the areas of the signature peptides from the tryptic digest alone to the areas obtained in the generation of the affinity purification external reference curve. For the $0.1,1,3$, and $5 \mu \mathrm{g}$ levels, the calculated percentage of affinity purified to digested areas are 49, 60, 65, and $69 \%(\mathrm{~m} / \mathrm{m})$, respectively, demonstrating a robust level of binding to allow meaningful interpretation of the results in the quantification analysis. 
Table 1. Quantification Results of Standard Addition

Analysis of CRP Serum Samples ${ }^{a}$

$\begin{array}{ccccc}\text { day } & \begin{array}{c}\text { >replicate } \\ (\mathrm{mg} / \mathrm{L})\end{array} & >\text { CRP mean } & \begin{array}{c}>\text { daily } \\ \text { mean }(\% \mathrm{CV})\end{array} & \text { > overall set } \\ 1 & \mathrm{~A} 1 & 32.9 & & \\ 1 & \mathrm{~A} 2 & 29.3 & & \\ 1 & \mathrm{~A} 3 & 35.7 & 33.0 \mathrm{mg} / \mathrm{L} & \\ 3 & \mathrm{~A} 4 & 32.8 & & \\ 3 & \mathrm{~A} 5 & 37.3 & & \\ 3 & \mathrm{~A} 6 & 31.5 & 34.6 \mathrm{mg} / \mathrm{L} & 33.3 \mathrm{mg} / \mathrm{L}(8.7 \%) \\ 4 & \mathrm{~B} 1 & 0.35 & & \\ 4 & \mathrm{~B} 2 & 0.34 & & 0.34 \mathrm{mg} / \mathrm{L}(4.5 \%) \\ 4 & \mathrm{~B} 3 & 0.32 & & \end{array}$

${ }^{a}$ The concentration determined for each individual replicate is reported also indicating the day of affinity purification in the study. The daily mean was calculated, and $t$-test analysis indicated no difference between the daily groups for Set A $(p=0.567)$. The overall mean was determined from the values of the replicates for the high (Set A, $n=6$ ) and low (Set B, $n=3$ ) levels of CRP in serum.

Quantification. The separate concentrations determined using the two peptides in the study, GYSIFSYATK (1136 Da) and EVQGEVFTKPQLWP (1820 Da), were not significantly different $(p>0.05)$, and the response areas of each peptide were combined for the analysis. The standard addition approach assumes that a linear relationship exists within the response at each level and $r^{2}$ values were obtained ranging from 0.9881 to 0.9995 and 0.9897 to 0.9967 for Sets A and B, respectively, considering the 0,1 , 2, $3 \mu \mathrm{g}$ levels. Quantification was performed using a one-point standard addition at the level of $3 \mu \mathrm{g}$. The results of standard addition LC-MS/MS measurements of high level of CRP in serum are presented in Table 1 with the concentration of 33.3 $\mathrm{mg} / \mathrm{L} \pm 8.7 \%$ (mean \pm coefficient of variance $(\mathrm{CV})$ ). The concentration of the low CRP sample was found to contain $0.34 \mathrm{mg} / \mathrm{L} \pm$ $4.5 \%$ also shown in Table 1.

As a means to confirm the results of the standard addition, the external reference approach was used as a second quantification method for CRP in serum. In this approach, external calibration solutions of CRP were affinity purified and digested under conditions identical to those of the serum samples to generate calibration curves for the replicates 1,2 , and 3 of both the high $\left(R^{2}=0.9898\right)$ and low $\left(r^{2}=0.9988\right)$ CRP serum sets. Average response factor calibration was used to assign values of CRP as $35.8 \pm 11.5 \%$ and $0.33 \pm 9.2 \%$, respectively, for the high and low CRP serum, which was not significantly different from the standard addition approach.

The expected values of the high and low CRP sera, as provided by the manufacturer, are based on immunoturbidimetric assays, and several values are provided according to the specific calibrant set which were employed. The total range of values for the high CRP serum was $28.79-45.85 \mathrm{mg} / \mathrm{L}$. The results for this material in the current study are well within the range of expected values. The total range of values for the low CRP serum as provided by the manufacturer was $0.39-0.69 \mathrm{mg} / \mathrm{L}$, which is slightly higher than the results from the current study. The values reported in this study agree closely with the values assigned by immunoassay. Reference measurement methods typically measure only a limited number of samples, usually the one to four levels which might be found in a typical reference material, and the two levels of CRP measured in the current study reflect this pattern. Comparison of the results with the immunoassay-value-assigned control serum was used for the assessment of the effectiveness of the method.

An additional analysis was performed using standard addition in serum without the use of affinity purification. CRP-spiked serum was directly digested to produce a measurement for the high serum sample with a mean $(n=5)$ of $32.7 \mathrm{mg} / \mathrm{L} \pm 9.0 \%$ which was not significantly different $(p=0.565)$ from that obtained with affinity purification. However, the value for the low CRP serum without affinity purification, with a mean $(n=2)$ of $1.9 \mathrm{mg} / \mathrm{L}$, substantially overestimated the value of CRP in the sample as compared to both the manufacturer supplied values and the affinity purification approach. Additionally, the analysis was performed with only one peptide, YEVQGEVFTKPQLWP, as the other peptide was not detected. A reference method would demand a more robust measurement achieved by using multiple quantification peptides. Similarity of the measurements in the high CRP serum sample with and without affinity purification indicates that the higher-order threshold limit for CRP without affinity purification is between $\approx 0.3 \mathrm{mg} / \mathrm{L}$ and $32 \mathrm{mg} / \mathrm{L}$. Therefore, in order to measure CRP in serum at either normal or moderately elevated levels using multiple quantification peptides, affinity purification or alternative enrichment strategies are necessary to provide sufficient precision and accuracy for use in a reference measurement procedure.

\section{DISCUSSION}

Presently, there is a lack of reference measurement procedures for the measurement of low abundance proteins in complex biological matrixes such as serum. There are practical limits to the direct utilization of mass spectrometry in this application, following enzymatic digestion and subsequent analysis of the product peptides, despite the high degree of selectivity and precision attained with these instruments. Failure to quantify low abundance proteins is principally due to the significant signal-tonoise degradation of the low abundance peptides because of the ion suppression effects of the peptides of more abundant proteins. A number of studies have described techniques to increase the signal-to-noise ratio of peptides from low abundance proteins by using immunodepletion ${ }^{20}$ and/or size exclusion chromatography ${ }^{21}$ to remove abundant proteins and have been successful in the reduction of both the detectable and relative quantification concentrations. Of particular note is the CRP measurement performed by Kuhn et al. ${ }^{21}$ which combined immunodepletion and size exclusion chromatography with a labeled peptide IDMS approach using four tryptic peptides, one of which, GYSIFSYATK, was also used in the current study. Kuhn et al. gauged their results with immunoassay measurements and obtained values which were varied in differences up to 5-fold. Williams and Muddiman ${ }^{22}$ recently published a study whereby they were able to perform peptide IDMS to measure CRP in serum using nanoflow-MS/MS without prior fractionation. Using only a single peptide, they compared the concentration of CRP in serum to an immunoassay

(20) Adkins, J. N.; Varnum, S. M.; Auberry, K. J.; Moore, R. J.; Angell, N. H.; Smith, R. D.; Springer, D. L.; Pounds, J. G. Mol. Cell. Proteomics 2002, 1, 947-955.

(21) Kuhn, E.; Wu, J.; Karl, J.; Liao, H.; Zolg, W.; Guild, B. Proteomics 2004, 4, 1175-1186.

(22) Williams, D. K., Jr.; Muddiman, D. C. J. Proteome Res. 2009, 8, 10851090 . 
and found a good correlation $\left(R^{2}=0.9708\right)$. However, the difference between the two methods was an average of 1 order of magnitude. It is clear from both of these studies that the use of IDMS to quantify proteins can potentially yield significantly different values than those obtained from immunometric methods. Therefore, for the use of IDMS in reference measurement procedures for protein analytes such as CRP, a measurement approach designed to eliminate or diminish measurement bias, such as what we have presented here, is necessary to ensure accuracy.

The greatest improvement in MS measurement sensitivity of clinically relevant proteins published to date has occurred using the technique of stable isotope standards and capture by antipeptide antibodies (SISCAPA) whereby synthetic stable isotope peptides are spiked into the digested material, most often after preliminary enrichment techniques, and used as internal standards for quantification. ${ }^{23,24}$ However, these techniques are not suitable for the assignment of values to reference materials. A reference measurement procedure must be critically investigated as to all potential sources of error, which must be identified, evaluated, and mitigated to the greatest extent possible. The purpose of the current study was to examine the feasibility of using affinity techniques to purify intact protein as the principle means to reduce the signal-to-noise component of peptides from serum low abundance proteins in a manner in which the sources of bias can be controlled. While both SISCAPA and intact protein affinity purification are able to enrich peptides of the digested target protein, they differ in the bias they each possess. SISCAPA functions by capturing the target peptides after digestion with quantification determined by comparing against spiked-in synthetic labeled peptides. The technique is quite robust in quantifying peptides, however, the relationship between the quantity of spiked-in peptides and the initial undigested protein is a significant source of bias. ${ }^{25}$ The direct correlation between protein and peptide concentration assumes complete digestion with no tryptic side reactions occurring, such as transpeptidation. ${ }^{26}$ To the extent that digestion is incomplete, bias will be present in the SISCAPA measurement without any means to make correction. CRP circulates as pentamer, and the effects of inconsistent denaturing can induce a profound variability in digestion. Affinity purification of intact protein using standard addition avoids this particular bias, as any spiked protein is added before purification and denaturing/ digestion will occur equivalently in the native and spiked protein thus avoiding bias.

Affinity-based techniques have previously been applied to the detection and quantification of CRP using competitive immunoas$\mathrm{say}^{27}$ while MALDI-MS analysis of affinity-purified proteins has previously been described for detection-identification ${ }^{28-30}$ and

(23) Anderson, N. L.; Anderson, N. G.; Haines, L. R.; Hardie, D. B.; Olafson, R. W.; Pearson, T. W. J. Proteome Res 2004, 3, 235-244.

(24) Whiteaker, J. R.; Zhao, L.; Zhang, H. Y.; Feng, L.-C.; Piening, B. D.; Anderson, L.; Paulovich, A. Anal. Biochem. 2007, 362, 44-54.

(25) Brun, V.; Dupuis, A.; Adrait, A.; Marcellin, M.; Thomas, D.; Court, M.; Vandenesch, F.; Garin, J. Mol. Cell. Prot. 2007, 6, 2139-2149.

(26) Schaefer, H.; Chamrad, D. C.; Marcus, K.; Reidegeld, K. A.; Bluggel, M.; Meyer, H. E. Proteomics 2005, 5, 846-852.

(27) Tsai, H. Y.; Hsu, C. F.; Chiu, I. W.; Bor Fuh, C. Anal. Chem. 2007, 79, 8416-8419.

(28) Kilpatrick, E. L.; Hildebrandt, J. D. J. Biol. Chem. 2007, 282, 14038-14047.

(29) Cole, J. R.; Dick, L. W., Jr.; Morgan, E. J.; McGown, L. B. Anal. Chem. 2007, 79, 273-279. structural ${ }^{31}$ purposes for proteins due to high mass accuracy. Efforts at quantification using MALDI-MS ${ }^{32,33}$ have been published but are inherently encumbered by the higher-order quantitative limitations. Nonetheless, these methods are often multiplexed and offer rapid, alternative approaches for the generation of lowerorder methods for more routine analyses. LC-MS/MS analysis has also been previously described in conjunction with affinity purification to identify proteins and associated modifications using stably expressed protein in cell lines. ${ }^{34}$

The use of affinity chromatography for both analyte enrichment and purification in the measurement approach can be a potential source of measurement bias and must be properly evaluated. If the monoclonal antibody is differentially selective for specific molecular forms of CRP, or if there are matrix effects that prevent equilibrium binding of CRP to the antibody, the amount of CRP captured for quantification after affinity chromatography will not be well correlated to the actual amount in the serum. One-point standard addition, in which a single known amount of CRP standard is added to the serum sample being measured, is effective in eliminating potential matrix effect bias. ${ }^{35}$ As the CRP standard used in this study was purified from human serum, the structural and molecular differences between CRP in the standard and samples will be minimized and offer the greatest affinity equivalency possible. If there is molecular and structural identity between the intrinsic serum CRP and the CRP added, any bias in the affinity chromatographic step should equally affect the intrinsic protein and the protein added for standard addition and thus be eliminated.

The approach taken in this study to measure peptides released from the protein through enzymatic digestion using trypsin in order to quantify the protein is well established. Two tryptic peptides were analyzed by LC-MS/MS in the current study to increase the confidence in the measurements obtained, particularly when, as in the current study, similar values are obtained with each peptide. The trypsin digestion process can often be inefficient, producing variably incomplete yields of tryptic peptides due to differences in sample matrix, protein structure, and specific amide bond conditions at each tryptic cleavage site within the same protein. Therefore, the trypsin digest can potentially be a significant source of measurement bias. The standard addition approach negates potential digest bias, as the magnitude of any partial trypsin digestion would be the same for the intrinsic serum CRP as for the standard spike. The inefficiency of the digestion process is still present in the current study; however, it is now applied equally to both the endogenous and spiked CRP, requiring only the single assumption that the spiked and sample proteins are proteolyzed similarly. CRP purified from human serum was used as the standard in order to minimize differences from the

(30) Nedelkov, D.; Nelson, R. W.; Kiernan, U. A.; Niederkofler, E. E.; Tubbs, K. A. FEBS Lett. 2003, 536, 130-134.

(31) Sparbier, K.; Asperger, A.; Resemann, A.; Kessler, I.; Koch, S.; Wenzel, T.; Stein, G.; Vorwerg, L; Suckau, D.; Kostrzewa, M. J. Biomol. Technol. 2007, $18,252-258$.

(32) Kiernan, U. A.; Addobbati, R.; Nedelkov, D.; Nelson, R. W. J. Proteome Res. 2006, 5, 1682-1687.

(33) Wang, K.-Y.; Chuang, S.-A.; Lin, P.-C.; Huang, L.-S.; Chen, S.-H.; Ouarda, S.; Pan, W.-H.; Lee, P.-Y.; Lin, C.-C.; Chen, Y.J. Anal. Chem. 2008, 80, 6159-6167.

(34) Wang, X.; Chen, C.-F.; Baker, P. R.; Chen, P.-L.; Kaiser, P.; Huang, L. Biochemistry 2007, 46, 3553-3565.

(35) Ellison, S. L. R.; Thompson, M. Analyst 2008, 133, 992-997. 
analyte. A second approach to evaluate bias was to use intact CRP protein as external calibrant in association with affinity purification. Different amounts of CRP in aqueous buffer were affinity purified concurrently with the standard addition samples. Both groups were digested at the same time using the same conditions. The CRP from the serum sample and the external calibrants were removed from their respective matrix, serum, and buffer, respectively, and were therefore digested in a new common matrix, namely, the elution buffer. Thus, any digestion bias present due to matrix effects on trypsin activity would be equivalent for calibrant and samples. This approach is only possible with the use of affinity chromatography.

In conclusion, the coupling of affinity purification with LC-MS/ MS does offer the ability to measure low abundance proteins in serum within acceptable limits and at a level which has previously been inaccessible to higher-order LC-MS/MS analysis. Additionally, results from the external reference calibration show the flexibility of this technique for other approaches, and the future development of intact isotopically labeled proteins would lend itself to the use of isotopic-dilution mass spectrometry analysis for the generation of higher-order quantification methods for CRP in serum. Current efforts are underway to generate ${ }^{15} \mathrm{~N}$-labeled CRP protein for use in future studies utilizing IDMS analysis and continued development of higher quantification methods incorporating additional signature peptides.

\section{ACKNOWLEDGMENT}

The authors acknowledge the assistance of Dr. Matthew J. Vergne in the development of LC-MS/MS methods. This work initially was presented as a poster at the annual meeting of the American Association of Clinical Chemistry on July 30, 2008, in Washington, DC.

\section{SUPPORTING INFORMATION AVAILABLE}

Additional information as noted in text. This material is available free of charge via the Internet at http://pubs.acs.org.

Received for review July 17, 2009. Accepted September 2, 2009.

AC901597H 